\title{
Magnitude of Malnutrition and Associated Factors Among Pregnancy and Lactating Mother in Meisso Health Center, Meisso Woreda, Oromia Region
}

\author{
Masresha Leta Serbesa ${ }^{1, *}$, Maleda Tefera Iffa ${ }^{2}$, Mohammed Geleto ${ }^{3}$ \\ ${ }^{1}$ Department of Midwifery, Harar Health Science College, Eastern Ethiopia, Harar, Ethiopia \\ ${ }^{2}$ Department of Nursing, Haramaya University, Eastern Ethiopia, Harar, Ethiopia \\ ${ }^{3}$ MEISSO HEALTH CENTER, Eastern Oromia Region, Ethiopia
}

Email address:

masreshaleta3@gmail.com (M. L. Serbesa), maledaifa.21@gmail.com (M. T. Iffa)

${ }^{*}$ Corresponding author

\section{To cite this article:}

Masresha Leta Serbesa, Maleda Tefera Iffa, Mohammed Geleto. Magnitude of Malnutrition and Associated Factors Among Pregnancy and Lactating Mother in Meisso Health Center, Meisso Woreda, Oromia Region. International Journal of Biomedical Science and Engineering. Vol. 7, No. 1, 2019, pp. 1-7. doi: 10.11648/j.ijbse.20190701.11

Received: March 3, 2019; Accepted: April 4, 2019; Published: May 27, 2019

\begin{abstract}
Introduction: - Malnutrition is one of the major nutritional problems in which the physical function of an individual is impaired to the point which cannot longer maintain adequate body performance process such as growth, physical work and resisting and recovery from the disease. Malnutrition usually stems from low economical situation, poor personal and environmental hygiene and ignorance are contribute to magnitude of high incidence of acute problem of malnutrition. Recent studies found that the center of the problems is the break ward socio economic development of the country which in turn results in one of the lowest standard of living, low level of social service, poor environmental conditions. In this respect it no disheartening to the not that the state of the health service in Ethiopia is low even when compared to the sub-Saharan Africa countries. Objective: To assess magnitude of malnutrition and associated factors among pregnancy and lactating mother in Mi'esso health center, Mi'essoworeda, Oromia Region, Ethiopia. Methods: Institutional based cross sectional study was employed. The study was conducted from Feb 1-May 30, 2017 among pregnancy and lactating in Mi'essoworeda. A total of 304 sample was selected by systematic random sampling from the list of Clients, with different socio-demographic and the collected data was analyses by using computer with the total budget of 4,846.9 Ethiopian birr. Result: The nutritional status of pregnancy and lactating mother based on their weight and height the BMI of the respondents indicates overweight accounts $12.6 \%$, underweight $30.3 \%$, and majority of the pregnancy and lactating mother accounts for $57.2 \%$ were normal nutritional level. The weight and height/ length of the pregnancy and lactating mother included in the study were between $1.56 \mathrm{~m}$ to $1.78 \mathrm{~m}$ and weight $53 \mathrm{~kg}$ to $84 \mathrm{~kg}$. From multiple logistic regression analysis family income (AOR=2.056, 95\% $\mathrm{CI}=1.051-4.023)$ and age of women $(\mathrm{AOR}=2.169(1.015-4.634)$ were significantly associated with the nutritional status of the study participants. Recommendation: Facilitate rural-urban community's access to information on nutrition like feeding practice sanitation, health and development program during pregnancy and lactate and efforts to expand awareness of women education using selected dietary during pregnancy and lactation.
\end{abstract}

Keywords: Magnitude, Pregnancy, Malnutrition

\section{Background}

The World Health Organization (WHO) defines malnutrition as "the cellular imbalance between supply of nutrients and energy and the body's demand for them to ensure growth, maintenance, and specific function. Contrary to the common use, the term malnutrition refers not only to deficiency states, but also to excess or imbalance in the intake of calories, proteins and/or other nutrient [1].

Adequate nutritional status of pregnant mothers is essential for their health and pregnancy outcomes. Due to increased 
nutritional requirements, pregnancy is a critical period for meeting the body's demand for macro and micronutrients. Thus, anemia and vitamin A deficiency (VAD) are common micronutrient deficiencies, which affecting 53.8 million pregnant women in the world [2].

Poor nutrition in pregnancy in combination with infections is a common cause of maternal and infant mortality and morbidity, low birth weight and intrauterine growth retardation (IUGR). Malnutrition remains one of the world's highest priority health issues, not only because its effects are so widespread and long lasting but also it can be eradicated best at the preventive stage [3].

All human beings need a balanced amount of nutrients for proper functioning of the body system. Nutrition is a fundamental pillar of human life, health and development throughout the entire life span [1]. Proper food and good nutrition are essential for survival, physical growth, mental development, performance and productivity, health and wellbeing. However, the nutrition requirement varies with respect to age, gender and during physiological changes such as pregnancy. Pregnancy is such a critical phase in a woman's life, when the expecting mother needs optimal nutrients of superior qualities to support the developing fetus.

Malnutrition manifests itself as a function of many and complex factor. It is directly linked to inadequacy at diet and diseases under living factors which include crisis in house hold food security, in appropriate child care and feeding practice, unhealthy place of residence and insufficient basic health services for residence for these more, poor socioeconomic variable, cultural belief and lacks of parents education, especially that of mothers are cited to affect a child national status [4].

Many women in Africa suffer from chronic energy deficiency, inadequate weight gain during pregnancy, and poor micronutrient status. Insufficient food intake, highenergy expenditure, micronutrient-deficient diets, infections, and the demands of pregnancy and lactation contribute to maternal malnutrition [5].

Maternal mortality is unacceptably high. About 800 women die from pregnancy- or childbirth related complications around the world every day [6]. In 2013, 289 000 women died during and following pregnancy and childbirth. Almost all of these deaths occurred in lowresource settings, and almost all maternal deaths (99\%) occur in developing countries. More than half of these deaths occur in sub-Saharan Africa [6]. According to Ethiopian Demographic Health Survey (EDHS) 2011, maternal mortality in Ethiopia was 676. Twenty percent of maternal deaths in Africa have been attributed to anemia [7]. In SubSaharan Africa, iron and folate deficiencies are the most common causes of anemia in pregnant women. Anemia has a variety of converging contributing factors but iron deficiency is the cause of $75 \%$ of anemia cases.

Nutrition during the periconceptional period is a key component of healthy pregnancy outcomes [8]. If there is maternal malnutrition on the pregnant mother, it was have consequences like: increased infection, anemia, decreased immune function, lethargy and weakness, low productivity, obstructed labor, high maternal mortality on the mother, and increased fetal and neonatal death, intrauterine growth retardation, low birth weight, preterm delivery, decreased immune function, birth defects, cretinism and decreased intelligent quotient (IQ) on the fetal side. It further affects the family and community socioeconomic conditions [9].

Maternal malnutrition is influenced not only by lack of adequate nutrition but also influenced by factors like social and psychological factors, nutritional knowledge of mothers and biological changes that influence perceptions of eating patterns during pregnancies [10].

In Ethiopia antenatal care (ANC) coverage by skilled provider in 2011 was $34 \%$. Prevalence of anemia among pregnant women was 22 , but only $16.8 \%$ of pregnant mothers had taken iron tablets during pregnancy [11]. Now-a-days, the Ethiopian government's commitment is above all times to improve the maternal and perinatal health in particular [12].

Many researches and projects focused on maternal health are common, but little attention is given to maternal nutrition in the study area [5]. It is clear that maternal nutrition is crucial in reducing maternal and infant morbidity and mortality but no study has been conducted to assess malnutrition and factor associating of pregnant and lactating mothers in the study area. So this study is aimed to assess the malnutrition and factor associating among pregnant and lactating mother attending ANC clinics in Meisso health center regarding the meaning, the importance and constituents of a well-balanced diet and practices of taking the necessary nutrients during pregnancy and lactating.

Therefore, This study designed to assess the crucial factors that addressed nutritional. Statuses of pregnancy and lactating mother were the most groves which affects nutritional problem. Providing important information about nutritional problem of the community is one of the major responsible tasks to the professional of the countries. This study was also help to: -Provide information for government and nongovernmental health organization that helps to work together on malnutrition. This survey was help monitoring and evaluation on nutrition program me in the area. Support concerned bodies (Health sector, Agriculture) for prevention of malnutrition.

The result obtained from this study can be used as a base line data for further similar or related studies. It may also be help to identify critical points on malnutrition in the Mi'essoworeda. The result of this study give useful ground for government agencies to intervene the practice of standard precaution and the factors hinders it; Generally, this study was used as base line information for further studies and it also help any organizations or individual who want to study on this issue.

\section{Method and Material}

An Institutional based cross sectional study was conducted to assess the magnitude of malnutrition and associated factors among pregnancy and lactating mothers in Mi'esso health 
center, Mi'esso woreda, Oromia Region, Ethiopia from March 1 to March 30, 2017 among 304 randomly selected pregnant and lactating mothers from Mi'esso health center from those pregnant and lactating women who are coming to Mi'esso health center during the study period. Based on the statistical abstract of Oromia Regional State of (2008), Western Hararghe is the fifth largest zone of the administrative zones of Oromia Regional State. It constitutes about $7 \%$ of the size of the region and covers $22,623 \mathrm{~km}^{2}$. The population and housing census on 2008 indicate that the Zone was projected to have a population of 2,739,390 (CSA, 2008).Average per month around 400 pregnant and 200-300 lactating women's visiting the health center.

Mi'essoWoreda is Kola climatic condition. Mi'essoWoreda has a population of 175,313 and about 93,604 peoples of Mi'essoWoreda is rural inhabitants (CSA, 2008). The mean annual temperature of the Woreda is about $24.5 \mathrm{OC}$ and the mean annual rainfall is about $1000 \mathrm{~mm}$. Mi'essoworeda and about $25 \mathrm{~km}$ away from Chiro town on the well-structured asphalt road.

Health service coverage of the region is $85 \%$. There are no hospital and four public health centers, and about 23 primary healthpost with 162 health care providers, in the mi'essoworeda.

Sample size was determined by using a single proportion sample size calculation formula with a source of population size greater than 10,000 and from previous studies done in Ethiopia indicates that the prevalence of malnutrition among pregnancy and lactating women is about $41 \%$ and, margin of Error $5 \%$ and $95 \%$ confidence interval and adding nonresponses rate of $10 \%$. The study participant were selected from those pregnant and lactating women who were coming for ANC and PNC service in the selected health institution during the study period.

The data was collected by using structured questionnaires which is adopted from different research done on related topic [3, 8]. The questionnaire contains close-ended and question including information on malnutrition and relating factors of malnutrition among pregnancy and lactating mothers including measurement of $\mathrm{Wt}, \mathrm{Ht}$ and calculating BMI. This questionnaire was prepared in English and translated to local language Afan Oromo with this some quality control measurement is practiced. The questionnaire was standardized, reliable and valid. Before actual data collection, the questionnaire was pre-tested in $5 \%$ of patients at near public health center in Oromia region, which was not selected for the study using randomly selected patients having a follow-up in ANC and PNC clinic. Before data collection, we were obtained voluntary oral consent from participants.

This helps to ensure the clarity, ordering, consistency and acceptability of the questionnaire. After this, the questionnaire was ready after necessary corrections and training was given for data collectors for 3 days at the time of data collection. During data collection, the questionnaire was checked for completeness on daily basis by the data collector themselves and the supervisors. The completed questionnaire was also rechecked by the principal investigators to maintain the quality of data.

After data collection, each questionnaire was checked for completeness, then coded and entered into Epi-info version 3.5.1 for cleaning, editing, and analysis. The results was presented in the form of tables, figures and text using frequencies and summary statistics such as mean, mode, standard deviation and percentage to describe the study population in relation to relevant variables.

Statistical Analysis: Binary logistic regressions was used to assess association between explanatory factors and outcome variables after adjusting for the effects of other variables. Odds ratio with $95 \%$ confidence interval and $\mathrm{P}$ value less than $5 \%$ were used to measure the degree of association between independent and dependent variables.

In order to confirm the ethical and legal standard of the investigator, approval was obtained from the ethical review board of Harar health science college. The survey was commence after written consent obtained from Mi'esso health center.

Operational Definition

Lactating mother: mother who was feeding breast milk for her infant/child during the study period.

Under nutrition-lactating mothers with BMI result of $\leq$ $18.5 \mathrm{~kg} / \mathrm{m}^{2}$.

Food secure households: Households those experience none of the food insecurity (access) conditions or just experience worry, but rarely in the past 4 weeks was labeled as 'food secured

\section{Result}

Socio demographic profile

Table 1. Enrolment and socio-Demographic Characteristics of pregnancy and lactating mothers in Mi'esso health center.

\begin{tabular}{llll}
\hline s/n & Respondents & Frequency & Percentage \\
\hline 1 & Pregnancy & 166 & $55 \%$ \\
2 & Lactating & 138 & $45 \%$ \\
& Total & 304 & $100 \%$ \\
1 & Resident & Urban & 178 \\
& & Rural & 126 \\
2 & Age & $<20$ years & 38 \\
& & $20-30$ year & 113 \\
& & $31-40$ year & 89 \\
& & $41-49$ year & 64 \\
& Educational Status & Primary $(1-8)$ & 150 \\
\end{tabular}




\begin{tabular}{llll}
\hline s/n & Respondents & Frequency & Percentage \\
\hline \multirow{3}{*}{5} & \multirow{2}{*}{ Religious } & Illiterate & 127 \\
& & Orthodox & 65 \\
& & Muslim & 217 \\
6 & \multirow{2}{*}{ Occupation } & Protestant & 22 \\
& & Farmer & 127 \\
& & Merchant & 82 \\
& & Civil Servant (employer) & 59 \\
7 & Family Size & Others & 36 \\
& & $<4$ & 216 \\
8 & Monthly income & $>4$ & 88 \\
& & $>1000$ & 143 \\
& & $1000-2000$ & 69 \\
9 & Latrine utilization & $>2000$ & 92 \\
& & Yes & 178 \\
10 & Source of drinking water & No & 126 \\
& & Pipe water & 178 \\
& & Un protected water & 209 \\
\hline
\end{tabular}

Knowledge of the respondents about nutrition

Table 2. Distribution of different facilities among the study pregnancy and lactating mother in Mi'esso health center.

\begin{tabular}{|c|c|c|c|c|}
\hline & Variables & Variables & Frequency & Percentage \\
\hline \multirow{3}{*}{1} & \multirow{2}{*}{ Know their nutritional status } & Yes & 51 & 16.62 \\
\hline & & No & 253 & 83.38 \\
\hline & \multirow{3}{*}{ Feeding style } & Vegetarian & 204 & $67.1 \%$ \\
\hline \multirow[t]{2}{*}{2} & & Meat and dairy with product & 67 & $22 \%$ \\
\hline & & Both equally & 33 & $10.9 \%$ \\
\hline \multirow{4}{*}{3} & \multirow{4}{*}{ Diet with 24 hours } & More than 3 times & 78 & $25.7 \%$ \\
\hline & & Three times & 130 & $42.8 \%$ \\
\hline & & Two times & 96 & $31.6 \%$ \\
\hline & & Total & 304 & 100 \\
\hline
\end{tabular}

Practices of mothers on maternal nutrition during pregnancy

Table 3. Nutritional practices of pregnant and lactating mothers attending Mi'esso health center.

\begin{tabular}{llllll}
\hline No. & Food group & Yes & \% & No & \% \\
\hline 1 & Following specific dietary regimen & 56 & 18.42 & 248 & 81.58 \\
2 & Using iodize salt to cook the main meal & 235 & 77.3 & 69 & 22.7 \\
3 & Habit of eating fresh citrus fruits/juice & 45 & 14.8 & 259 & 85.2 \\
4 & Eating fruits and vegetables like tomato, onion, & 95 & 31.3 & 209 & 68.8 \\
5 & Iron Supply & 76 & 25 & 228 & 75 \\
6 & Frequency of meal per day more than usual & 83 & 27.30 & 221 & 72.70 \\
7 & Habit of eating more carbohydrates & 73 & 24 & 231 & 76 \\
8 & Eating protein such as meat, milk and milk product daily & 129 & 42.4 & 175 & 57.6 \\
\hline
\end{tabular}

Associated Factor of nutritional status of pregnant and lactating mothers

To determine the association between nutritional status and variables bivariate analysis was performed using logistic regression. The result indicates that there is association between nutritional status of pregnant and lactating mothers.
As can be seen from the below table family income, habit of eating daily protein containing food and ages of mothers were found to be associated with nutritional status of pregnant and lactating mother.

Table 4. Bivariate logistic regression test of nutritional status of lactating mothers with selected variables.

\begin{tabular}{|c|c|c|c|c|c|}
\hline \multirow{2}{*}{ Variable } & \multirow{2}{*}{ Categories } & \multicolumn{2}{|l|}{ BMI } & \multirow{2}{*}{ P-value } & \multirow{2}{*}{$\operatorname{COR}(95 \% \mathrm{CI})$} \\
\hline & & Underweight (>18.5) & Normal weight $(\geq 18.5)$ & & \\
\hline \multirow{3}{*}{ Monthly income } & $>1000$ & $73(79.35 \%)$ & $71(33.49 \%)$ & 0.722 & $0.827(0.290-2.456)$ \\
\hline & $1001-2000$ & $17(18.47 \%)$ & $51(24.05 \%)$ & 0.000 & $0.126(0.48-0.330)$ \\
\hline & $<2000$ & $2(2.17)$ & $90(45.28 \%)$ & & \\
\hline \multirow{2}{*}{ Eat protein daily } & Yes & $13(14.13)$ & $116(54.72 \%)$ & & \\
\hline & No & $79(85.87 \%)$ & $96(31.57 \%)$ & 0.049 & $0.045(0.002-0.986)$ \\
\hline \multirow{3}{*}{ Age } & $<20$ & $12(13 \%)$ & $26(12.26 \%)$ & 0.052 & \\
\hline & $31-40$ & $40(43.5 \%)$ & $49(23.12 \%)$ & 0.006 & $0.244(0.089-0.668)$ \\
\hline & $41-49$ & $21(22.8 \%)$ & $43(20.28 \%)$ & 0.116 & $0.452(0.168-1.218)$ \\
\hline
\end{tabular}


Monthly income of pregnant and lactating mothers are strongly associated with nutritional status $(\mathrm{p}<0.001)$. Whereas variables like occupation, marital status, educational status, culture, religion, and family size have no association with nutritional status of pregnant and lactating mothers $(\mathrm{p}>0.05)$. Family income is factor found to affect the nutritional status. Family of pregnant and lactate mothers those who got monthly income 1001-2000 birr were less likely exposed underweight than those who got less than $<1000$ birr $(\mathrm{COR}=0.827 \% \mathrm{CI}=0.290-2.356)$ and $(\mathrm{COR}=0.126$, $95 \% \mathrm{CI}=0.048-0.330)$ respectively. Habit of eating daily protein containing food of pregnant and lactate mothers were another factor that affects nutritional status. Those mothers who have no daily habit of eating protein containing food were more likely exposed to underweight than who have daily habit eating protein containing food
$(\mathrm{COR}=0.45 \% \mathrm{CI}=0.002-0.986)$.

After bivariate analysis, multivariate analysis of logistic regression was performed to filter the net effect of each independent variable that associate in the bivariate model analysis with nutritional status of pregnant and lactate mothers by controlling the other independent variables in table 3 . In a multivariate logistic regression analysis family income and age found to have significant association with nutritional status of lactate mothers. Concerning family income, those pregnant and lactating mothers earn monthly income greater than 2000 birr were less likely to be under weight than those lactating mothers who got less than 1000 birr $(\mathrm{AOR}=2.056,95 \% \mathrm{CI}=1.051-4.023)$. Those lactate mothers who are age between 21-30 year were less likely to be under weight than those mothers who are age greater than $30(\mathrm{AOR}=2.172,95 \% \mathrm{CI}=0.843-5.598)$

Table 5. Multiple logistic regression of nutritional status of lactate mother.

\begin{tabular}{|c|c|c|c|c|c|}
\hline \multirow{2}{*}{ Variable } & \multirow{2}{*}{ Categories } & \multicolumn{2}{|l|}{ BMI } & \multirow{2}{*}{ P-value } & \multirow{2}{*}{$\operatorname{AOR}(95 \% C I)$} \\
\hline & & Underweight (>18.5) & Normal weight $(\geq 18.5)$ & & \\
\hline \multirow{4}{*}{ Monthly income } & $>1000$ & $73(79.35 \%)$ & $71(33.49 \%)$ & 0.035 & $2.056(1.051-4.023)$ \\
\hline & $1001-2000$ & $17(18.47 \%)$ & $51(24.05 \%)$ & 0.001 & $5.632(2.694-11.777)$ \\
\hline & $<2000$ & $2(2.17)$ & $90(42.46 \%)$ & & \\
\hline & Total & 92 & 212 & & \\
\hline \multirow{4}{*}{ Age } & $<20$ & $12(13 \%)$ & $26(12.26 \%)$ & & \\
\hline & $21-30$ & $19(20.7 \%)$ & $94(44.34 \%)$ & 0.108 & $2.172(0.843-5.598)$ \\
\hline & $41-49$ & $21(22.8 \%)$ & $43(20.28 \%)$ & 0.638 & $1.474(0.665-3.267)$ \\
\hline & Total & 92 & 212 & & \\
\hline
\end{tabular}

\section{Discussion}

This study reports on the magnitude of malnutrition among pregnancy and lactating mothers shows their demographic characteristics on the nutritional status of pregnancy and lactating mother in Mi'esso health center on 304 pregnancy and lactating mother representing the health center. The study may be regarded as a reasonable reflection of the nutritional status of pregnancy and lactating mother. A number of similar studies have been conducted in varies part of the countries.

Different studies show that education has prominent effects on nutritional status of pregnancy and lactating mothers. Some of the respondents were illiterates the result shows 127 (41.8\%) no education, primary $150(49.3 \%)$ the impact of family education is not only through its effect on nutrition but also through additional income and the pregnancy and lactating mother ability to make better decisions for herself and next for her child. The educational level of the pregnancy and lactating mother in line with study showed the highest impact on the adolescents' health related dietary habits since they cook family meal. Mostly educated pregnancy and lactating mother are cautious of what theyeat than uneducated pregnancy and lactating mother. Study in urban slums of Dibrugarah showed that prevalence of mal nitrated mothers was much higher among low incomes and whose mother $\mathrm{s}$ wasilliterate and a significant (Himashree Bhattacharyya and AlakBarua, 2013).
According to result the large family size seen in the area was one of the factors affecting the nutritional status of pregnancy and lactating mothers. The reason is that no enough time for proper care for their pregnancy and lactating mother and feeding and may increase the risk factor of mal nutrition. A larger family size associated with an increased risk of prevalence of malnutrition. The effect of a large family size with overcrowding and inadequate spacing has been implicated as a risk factor for prevalence of malnutrition, the results shows 5-2 family size $216(71.1 \%)$, less than four family size $88(28.9 \%)$ family size greater than four from 304 pregnancy and lactating mother.

The result of this study showed that pregnancy and lactating mother with prevalence of malnutrition lived in a household with low monthly income family.

Which were showed birr less than 1000 birr per month 143 (47\%), 1001-2000 birr per month 69 (22.7\%), and $92(30.3 \%)$ greater than 2000 birr per month for from 304 pregnancy and lactating mother.

Monthly income and attitude during pregnancy were identified as important predictors of knowledge of women on nutrition during pregnancy and lactation among the study participants. Similar study conducted in Wollega showed that educational level and monthly income were significantly associated with maternal nutritional knowledge [13].

According to response towards use of feeding style they show that $45(14.8 \%)$ had the habit of eating fresh citrus fruits juice, most of women, $67(22 \%)$ of women had the 
habit of taking protein daily and 129 (42.4\%) of pregnancy and lactation mothers practiced daily using of milk and milk products. As regards fresh vegetable, only 95 (25\%) of respondents practiced daily. In the studies conducted in U.S. and Australia on adolescent it is observed that had inadequate consumption habits of fruits and vegetable, low intake levels of vitamins, calcium and, phosphorus and iron- rich foods.

Looking the percentage of overweight accounts $22.5 \%$, underweight $30.3 \%$, and majority of the pregnancy and lactating mother accounts for $47.2 \%$ were normal nutritional level. The weight and height/ length of the pregnancy and lactating mother included in the study. Of which study conducted in rural community of Tigray region indicated that about $25.5 \%$ and $58.3 \%$ of them were stunted and thin respectively. Similarly, the study made to identify on socioeconomic factors associated with underweight and stunting among adolescents of Jimma Zone, identified that most of the underweight adolescents $(53.20 \%)$ were females (HuruyAssefa, 2010). According to the recent Demographic and Health Survey of Ethiopia (EDHS), about 27\% of women age 15-49 are thin 9\% are moderately or severely thin and only $6 \%$ of women are overweight or obese (EDHS, 2011).

\section{Conclusion}

Education is one of the most important resources that enable family to provided appropriate care for their pregnancy and lactating mother, which is an important determinant of pregnancy and lactating mother's growth and development. The risk of prevalence is increased when the monthly income is lower than 50 united state dollars USD below 1000 Ethiopian birr. According to result the large family size seen in the area was one of the factors affecting the nutritional status of pregnancy and lactating mother in the family. The reason that family not gets enough time for proper female care and feeding and may increase the risk factor of mal nutrition. A larger family size associated with an increased risk of prevalence of stunting. This study found evidence that socio economic and demographic variables have a significant influence on the nutritional status of pregnancy and lactating mother. Therefore need to take action its starting point and address those problems, and seeing that these strategies are implemented in a way that works.

This study also showed that most of mothers had a poor level of knowledge and practices about nutrition during pregnancy and lactation. Furthermore, the most significant predicting factors for knowledge in this study were high women education followed by monthly income and attitude. Monthly income and occupation were predicting factors for nutritional practices during pregnancy and lactating. So, it is obvious that good knowledge about maternal nutrition usually resulting in good dietary practices which are important for health of the mother and the fetus.

\section{Recommendation}

Depending on the result of the study area in order to prevent this nutritional problem that happened among pregnancy and lactating mother's formulated the following recommendation:-

1. Facilitate rural-urban community's access to information on nutrition like feeding practices sanitation, health and development programs.

2. Efforts to expand awareness of women education using selected dietary during pregnancy and lactation

3. Government and other stockholders needs to develop educational programs about nutritional problem identification and solution especially community education about particular nutritional needs to pregnancy and lactating mother and community needed to know how to address main infectious disease.

4. Family care to their pregnancy and lactating mother should actively work

\section{List of Acronyms and Abbreviation}

CSA: Central Statistical Authority, DHS: Demographic Health Service, EJHD: Ethiopia Journal of Health Development, IDD: Iodine Deficiency Disorder, IES: Information Education Communication, MUAC: Middle Upper Arm Circumference, NGO: Non Governmental Organizations, PEM: Protein Energy Mal nutrition, VAD: Vitamin "A" Deficiency, WHO: World Health Organization

\section{References}

[1] Bank W. Repositioning Nutrition as central to development: A strategy for large scale action, The International Bank for Reconstruction and Development, World Bank, Washington DC, USA. 2006.

[2] Latifa MF MH, Nihal SS (2012) Nutritional Awareness of Women during Pregnancy. J American Sci 2012.

[3] Daba G BF, Fekadu H, Garoma W. i. 2013; 3 (235). Assessment of Knowledge of Pregnant Mothers on Maternal Nutrition and Associated Factors in Guto Gida Woreda, East Wollega Zone, Ethiopia. J Nutr Food Sci. 2013; 3.

[4] Ronsmans C GW. Maternal mortality: who, when, where, and why. The Lancet. 2014; 368: 1189-200.

[5] Berhan Y BA. commentary: acttions in the pipeline and the way forward to reduce maternal and perinatal mortality in Ethiopia. Ethiop J Health Sci. 2014: 149-68.

[6] Idowu O MC, Dopu S.. Anaemia in pregnancy: a survey of pregnant women in Abeokuta, Nigeria. African health sciences. 2007; 5: 295-9.

[7] Central Statistics Agency, 2011. Ethiopian Demographic and Health Survey, Addis Ababa, Ethiopia. 2011.

[8] Zhao A, Zhang, Y., Li, B., Wang, P., Li, J., Xue, Y. and Gao, H.. Prevalence of Anemia and Its Risk Factors Among Lactating Mothers in Myanmar. American Journal ofTropical Medicine and Hygine. 2014; 90: 963-7. 
[9] Ronsmans C GW. Maternal mortality: who, when, where, and why. The Lancet 2014; 368: 1189-200.

[10] Temesgen Desisa DW, Habtamu Fikadu and Dunkana Negussa. Nutritional Status and Associated Factors Among Lactating Mothers in Nekemte Referral Hospital and Health Centers, Ethiopia. International Journal of Nutrition and Food Sciences. 2015; 4: 216-22.

[11] Wang Z, Dang, S. and Yan, H.. Nutrient intakes of rural Tibetan mothers: a crosssectional survey. BMC Public Health. $2010 ; 10$.
[12] Kedir Teji OC, T. P., Tefera Belachew and O'Brien, N. M.. Seasonal variation in nutritional status and anemia among lactating mothers in two agro-ecological zones of rural Ethiopia: A longitudinal study. Elsevier Inc. 2015; 31: 1213-8.

[13] Daba G BF, Fekadu H, Garoma W. Assessment of Knowledge of Pregnant Mothers on Maternal Nutrition and Associated Factors in Guto Gida Woreda, East Wollega Zone, Ethiopia. J Nutr Food Sci. 2013; 3. 